\title{
ACUPUNTURA EN DERMATITIS ATÓPICA Y MECANISMOS NEUROINMUMONOLÓGicos. ACTUALIZACión
}

\author{
Martha P. Robayo M. M.D. ${ }^{1}$, Mauricio Ángel M. M.D. ${ }^{2}$ Y Claudia L. Robayo M. M.D. ${ }^{* *}$ \\ ${ }^{1}$ Dermatóloga Coordinadora Servicio de Dermatología. Hospital Militar Central. Bogotá, Colombia. \\ ${ }^{2}$ Magister en Medicina Alternativa - Medicina Tradicional China (MTCh) y Acupuntura Universidad Nacional de Colombia. \\ Asesor y conferencista invitado del área de MTCh y Acupuntura Universidad Nacional de Colombia. Bogotá, Colombia. \\ ${ }^{3}$ Estudiante Cohorte III Maestría en Medicina Alternativa línea de profundización Medicina Tradicional China y Acupuntura. \\ Universidad Nacional de Colombia, Bogotá, Colombia.
}

\begin{abstract}
Resumen
La dermatitis atópica es una patología de alta prevalencia tanto en nuestro medio como en el resto del mundo. Su etiopatogenia es compleja y multifactorial e involucra factores genéticos, ambientales e inmunológicos. Se han planteado múltiples opciones terapéuticas para su manejo y en años recientes se ha observado un incremento en el uso de la acupuntura como una opción que buscan los pacientes con patologías dermatológicas, en particular la dermatitis atópica. Estudios a nivel neuroinmunológico se han llevado a cabo para explicar los posibles mecanismos subyacentes involucrados en el efecto de este tratamiento. En este artículo se presenta una revisión sobre los aspectos más relevantes de esta entidad.
\end{abstract}

Palabras clave: acupuntura, dermatitis atópica, fisiopatología, inmunomodulación.

\section{ACUPUNCTURE IN ATOPIC DERMATITIS AND NEUROIMMUNOLOGY MECHANISMS. UPDATE}

\begin{abstract}
Atopic dermatitis isa high-prevalencediseasein our countryand the rest of the world. Its etiopathology is complex and multifactorial involving genetic, environmental and immunological factors. Many therapeutic options have been developed for its management. In recent years an increase in the use of acupunctureby patients with skin diseases, including atopic dermatitis, has been documented. Neuro-immunological studies have been conducted to explain the possible underlying mechanisms involved in the effect of this treatment.This articlepresentsa review ofthe most important aspectsof this issue.
\end{abstract}

Key words: acupuncture, dermatitis atopic, physiopathology, immunomodulation.

\section{ACUPUNTURA NA DERMATITE ATÓPICA E MECANISMOS NEUROINMUMONOLÓGICOS. Atualização}

\section{Resumo}

\begin{abstract}
A dermatite atópica é uma doença de alta prevalência em nosso país eo resto do mundo. Sua etiopatogênese é complexa e multifatorial e envolve fatores genéticos, ambientais e imunológicos. Têm sido levantadas muitas opções de tratamento para a sua abordagem e nos últimos anos têm visto um aumento no uso da acupuntura como uma opção para pacientes que procuram com doenças de pele, incluindo dermatite atópica. Estudos de nível Neuroimmunological têm sido realizados para explicar os possíveis mecanismos subjacentes envolvidos no efeito deste tratamento. Este artigo apresenta uma revisão dos aspectos mais importantes desta entidade.
\end{abstract}

Palabras-chave: acupuntura, dermatite atópica, fisiopatologia, imunomodulação

\section{Recibido: Mayo 20 de 2011 Aceptado: Junio 22 de 2011}

* Correspondencia: Claudia L. Robayo M. claudiaroba@hotmail.com. Dirección postal: Tr. 3 No.49-00. Facultad de Medicina. Universidad Militar" Nueva Granada", Bogotá, Colombia. 


\section{Introducción}

El uso de la acupuntura, tratamiento médico ancestral procedente de Asia, se ha incrementado día a día por parte de pacientes con trastornos dermatológicos crónicos incluyendo la dermatitis atópica (DA). En años recientes numerosos estudios se han llevados a cabo, para explicar los mecanismos subyacentes detrás del efecto terapéutico de la acupuntura en el tratamiento en algunos síntomas y patologías. En esta revisión se presentan los aspectos más relevantes de la DA así como evidencia emergente sobre los posibles mecanismos inmunomoduladores detrás del uso de la acupuntura en esta patología.

\section{¿Qué es la dermatitis atópica?}

La DA es una condición difícil de definir debido a sus características clínicas variables pero podría ser definida como una dermatosis inflamatoria, crónica, pruriginosa y recurrente $(1,2)$. Clínicamente se caracteriza por la presencia de prurito intenso, xerosis, lesiones tipo pápulas (ocasionalmente vesículas en niños) escoriadas y liquenificadas de distribución simétrica en zonas de flexión, curso intermitente e historia personal o familiar de otras condiciones atópicas $(1,3)$. La DA junto con el asma bronquial y la rinoconjuntivitis constituyen la triada atópica, siendo la DA la primera de las manifestaciones del proceso conocido como marcha atópica (4).

\section{Epidemiología}

La DA es una patología frecuente en la población. Se presenta principalmente en la infancia y la severidad del cuadro clínico disminuye con la edad (1). Alrededor del 50 $\%$ de los casos son diagnosticados en el primer año de vida y un $80 \%$ hasta los cinco años (4).

La prevalencia de la enfermedad ha aumentado en los últimos años y varia entre $0,73 \%$ en Tanzania a $23 \%$ en Noruega; en Colombia Dennis y col. (5) reportaron una prevalencia de 3,9\% y el estudio ISSAC (The International Study of Asthma and Allergies in Childhood) fase III encontró una prevalencia de $14,65 \%$ en niños de seis a siete años de edad y $12,28 \%$ en los trece a catorce años (6). La prevalencia ha aumentado de dos a tres veces en los países mas desarrollados, pero permanece baja en las regiones agrícolas de China, Europa del Este y África rural (6). La DA es más frecuente en familias pequeñas, en los primeros hijos y mejores condiciones económicas (4).

\section{Etiología}

La etiología de los procesos que desencadena la DA son complejos y multifactoriales, incluyen componentes genéticos, medioambientales y alteraciones inmunes (7).

\section{Factores Genéticos}

La importancia de los factores genéticos dentro de la patogénesis de la DA se evidencia por el aumento del riesgo para desarrollar la enfermedad cuando existe historia familiar de DA, principalmente los hijos de madres atópicas y la concordancia en la incidencia de la enfermedad entre gemelos monocigotos $(1,8)$. Se han reportado al menos tres grupos de genes que codifican proteínas estructurales, proteasas epidérmicas e inhibidores de proteasas generando alteración en la función de barrera cutánea e incrementando el riesgo de aparición de DA (8).

El gen que codifica la filagrina (proteína estructural del estrato córneo) localizado en el cromosoma 1q21 presenta mutaciones en el caso de la DA $(2,9)$; la alteración en la filagrina genera un incremento en el pH del estrato córneo alterando la función de la barrera cutánea, característica de la DA $(9,10)$.

\section{Factores Ambientales}

Los principales factores ambientales relacionados con la DA son: la polución y los microorganismos (1). La relación de la contaminación ambiental aun no es clara pero no se descarta, debido al aumento de la DA en las últimas décadas y su mayor prevalencia en áreas industrializadas (6). Con relación a los microorganismos se plantea la "hipótesis higienista" que sugiere, que la exposición tardía o escasa a microorganismos, retarda la maduración del sistema inmune promoviendo alteraciones de la IgE, relacionas con $\mathrm{DA}$, mientras que una exposición temprana a microorganismos promueve la maduración del sistema inmune (1).

Los pacientes con DA exhiben un riesgo aumentado a la colonización e infección por cierto tipo de microorganismos como Staphylococcus aureus, virus herpes y Malassezia sympodialis $(11,12)$ que el resto de la población. La colonización de la piel con $S$. aureus, es muy común en DA y el patógeno puede ser aislado entre un $80 \%$ a $100 \%$ de las lesiones atópicas, muchas de ellas sin signos clínicos de infección (13). El S. aureus en particular secreta varias exotoxinas que actúan como superantígenos para los linfocitos T y macrófagos desencadenando liberación de citoquinas y mediadores de la inflamación, estimulando los macrófagos 
epidérmicos y las células de Langerhans contribuyendo a la persistencia y exacerbación de la DA $(7,14,15)$.

\section{Factores Inmunológicos}

La patogénesis más ampliamente aceptada de la DA es el desbalance en la respuesta Th2/Th1, con incremento de los niveles de $\mathrm{IgE}$, eosinofilia e hiperreactividad de los mastocitos $(4,8)$.

\section{Fisiopatología}

La fisiopatología de la DA involucra complejas interacciones entre susceptibilidad genética (genes de la filagrina), factores inmunológicos (IgE, eosinófilos, desbalance de la respuesta Th2/Th1, citoquinas, mastocitos), defectos de la barrera cutánea, infecciones, factores neuroendocrinos (neurotrofinas) y factores ambientales (cambios climáticos, comida, aeroalergenos) (16). La patogénesis de la DA es atribuida a una anormalidad primaria del sistema inmune innato y adaptativo (17), generando una cascada de eventos con la producción y liberación de mediadores de la inflamación siendo los principales implicados las células T CD4 y CD8 $(18,19)$.

Por estímulos antigénicos, en la fase inicial hay activación preferencial de los linfocitos Th2 y las células Natural Killer (NK), con liberación de las citoquinas IL-4, IL-5, IL-13 y poca estimulación para interferón alfa (INF- $\alpha$ ) e IL-12. La IL-4 e IL-13 están implicadas en la fase inicial de la inflamación tisular y la IL-5 parece aumentar la sobrevida de los eosinófilos $(4,20,21)$. También se conoce que los monocitos de pacientes atópicos tienen mayor supervivencia y aumento en la producción de prostaglandina $\mathrm{E}$ y de IL-10 (reguladora del equilibrio entre la respuesta Th1 y Th2) que al parecer inhiben la respuesta Th1 y acentúa la secreción de IL-4 por parte de los Th2 (22).

La fase crónica se asocia a respuesta Th1 y Th0 con liberación de IL-5, factor estimulante del crecimiento de granulocitos (G-CSF), IL-11, IL-12, IL-18 e INF- $\alpha(4,9)$. Se ha observado que la sustancia $\mathrm{P}$ (neuropéptido) se encuentra involucrado en la activación y regulación de la respuesta inmune innata y la adaptativa y sus niveles están aumentados en piel y en sangre de los pacientes que padecen DA, así como alteraciones en las células dendríticas (células presentadoras de antígeno: APC) especialmente las células de Langerhans y las células dendríticas epidérmicas inflamatorias $(6,23,24)$.

Wuthrich refirió que la DA podría clasificarse en dos formas: una variable extrínseca que afecta aproximadamente entre el 70 y $80 \%$ de los pacientes adultos, donde se evidencia elevación importante de los niveles IgE en suero y una forma intrínseca que se observa en el resto de los casos con bajos niveles de IgE. Esta clasificación sugiere que la elevación de la IgE especifica no es un prerrequisito en la patogénesis de todas las formas de DA (22).

Además se ha observado un defecto en la función de la barrera epidérmica y se han postulado varios mecanismos como: alteración en las ceramidas, colesterol y ácidos grasos libres del estrato córneo causando xerosis $(9,25)$, que lleva al rascado, originando trauma mecánico de los queratinocitos y liberación de citoquinas proinflamatorias (4); también se evidencia que los queratinocitos de los pacientes con DA presentan un defecto intrínseco, relacionado con la producción en menor cantidad de péptidos antimicrobianos $(26,27)$, generando una disfunción en las primeras líneas de defensa de la piel, alteraciones en el $\mathrm{pH}$ del estrato córneo y cambios en la filagrina $(6,26)$. Los neuropéptidos y neurotrofinas modulan diferentes acciones y tiene una relación cercana con las células mastocíticas y células de Langerhans sugiriendo una conexión entre el sistema inmune y el sistema nervioso (6).

\section{Manifestaciones clínicas}

Las manifestaciones clínicas varían según la edad del paciente y el estadio en el que se encuentre la enfermedad. Según el tiempo de evolución se clasifica en: Fase aguda: presencia de pápulas-vesículas sobre una base eritematosa $(4,8)$. Fase subaguda: pápulas confluentes dentro de placas eritematodescamativas mal definidas $(4,8)$. Fase crónica: liquenificacion de las lesiones $(4,8)$.

Según la edad del paciente se clasifica en: Infancia: lesiones eczematosas papulo-vesiculares en cuero cabelludo y mejillas (8). Niñez: típicamente se observa compromiso de áreas flexoras y xerosis (8). Adolescencia y adultez: placas liquenificadas en áreas de flexión, cara y cuello (8).

Otra de las consideraciones que se observar a la hora del diagnóstico de DA es la denominada "personalidad atópica" caracterizada por: hiperquinesia, irritabilidad, ansiedad y depresión, rasgos a tener en cuenta al momento de realizar un tratamiento integral $(4,28)$.

\section{Criterios diagnósticos}

El tiempo de evolución, la morfología y la distribución de las lesiones ha hecho difícil definir criterios diagnósticos únicos. En 1980 Hanifin y Rajka establecieron los primeros criterios diagnósticos y que aun se utilizan; en 1994 se esta- 
blecieron los llamados criterios del Reino Unido los cuales tienen una especificidad del $92,8 \%$ y una sensibilidad del $87,9 \%$. Estos criterios se dividen en un criterio mayor único: prurito y cinco criterios menores:

1. Historia de personal o familiar de atopia

2. Historia de compromiso de pliegues cutáneos (antecubital, fosas poplíteas, tobillos o alrededor del cuello, mejillas en niños menores de diez años)

3. Historia de piel seca en el último año

4. Eczema flexural visible o eczema que afecta las mejillas, frente, extremidades dístales en menores de cuatro años

5. Inicio de la enfermedad antes de los dos años.

Para hacer el diagnóstico el paciente debe presentar el criterio mayor único y tres de los criterios menores (4).

\section{Tratamiento}

La meta del tratamiento de la DA es el control de los síntomas y no la curación de la enfermedad (29). Actualmente existen diferentes tratamientos disponibles para su manejo pero la falta de adherencia a los mismos, es la causa más común que induce a la falla terapéutica (30); la no adherencia es producto del temor a los eventos adversos de los medicamentos y a la prolongada duración del tratamiento. El estudio realizado por Aubert-Wastiaux y col (31) mostró que el $80 \%$ de los pacientes con DA, manifiesta tener temor al uso de corticosteroides por sus efectos a largo plazo.

En términos generales se emplean hidratantes y emolientes, corticoides tópicos y sistémicos, fototerapia, probióticos, inmunomoduladores, antihistamínicos, cambios en la dieta y medidas de aseo. Debido a la alteración en la calidad de vida de los pacientes y sus familiares es importante realizar un manejo conjunto que involucre tanto al cuidador, al paciente y un grupo interdisciplinario (32).

Teniendo en cuenta las consideraciones expuestas anteriormente, se justifica la búsqueda de opciones terapéuticas adicionales cuya efectividad sea demostrada con evidencia científica y que sean seguras para complementar el tratamiento de la DA. Dentro de estas opciones se plantea el uso de la acupuntura y la medicina tradicional China como herramienta coadyuvante.

A partir de la declaración de Alma Ata en 1978, donde se menciona el rol de los practicantes de medicinas tradicionales en el cuidado de la salud en atención primaria, la Organización Mundial de la Salud (OMS) empezó a prestar atención a la medicina tradicional. Casi tres décadas mas tarde, en la quincuagésima novena asamblea mundial de salud celebrada en Genova 2006, los 192 estados miembros fueron impulsados a promover la integración de la medicina tradicional en sus sistemas de salud pública y promover el diálogo académico con la medicina occidental moderna (33).

\section{Medicina tradicional China y acupuntura}

La medicina tradicional China (MTCh) se define como un sistema médico originario de China, caracterizado por su aproximación holística y el tratamiento basado en la identificación de patrones/diferenciación sindromática, que incluye medicina herbal, acupuntura, moxibustión y otras terapias no farmacológicas (33).

La acupuntura, como importante componente de la MTCh, originada hace más de 2500 años atrás, ha llegado ha ser un método terapéutico global debido a su eficacia y baja proporción de efectos adversos (34). Consiste en la estimulación con agujas filiformes de puntos predeterminados sobre el cuerpo (361 puntos y extraordinarios) llamados acupuntos, algunas veces usando calor (moxibustión), presión (acupresión) o electricidad (electroacupuntura: EA).

La teoría ancestral de la acupuntura promulga que el Qi o "Energía Vital" fluye a través de los meridianos, que son canales o vías que comunican los diferentes órganos y estructuras en el cuerpo. Según esta teoría la enfermedad ocurre cuando los meridianos son bloqueados sea por factores externos medioambientales o factores internos propios del individuo. La acupuntura genera un desbloqueo permitiendo el flujo normal de Qi restaurando la salud. La idea del Qi no ha sido sustentada por el conocimiento científico vigente (35).

Estudios imagenológicos y fisiológicos indican que la acupuntura activa el sistema nervioso central e induce procesos de inmunomodulación (35). Tradicionalmente la acupuntura ha sido usada para tratar diferentes síntomas y enfermedades tales como disfunciones del sistema nervioso autónomo, dolor, trastornos metabólicos y enfermedades del sistema nervioso central y periférico así como desordenes alérgicos entre otros (36).

\section{Mecanismos de acción de la acupuntura}

La inserción de agujas dentro del cuerpo activa fibras nerviosas aferentes, su señal viaja hasta la médula espinal y desde allí al sistema nervioso central, donde interviene en la síntesis y función de numerosos neuromoduladores $(36,37)$, induciendo la plasticidad en diferentes conexiones 
y rutas neuronales (38). Se ha propuesto que el efecto de la acupuntura esta correlacionado con la activación de las fibras nerviosas sensoriales tipo A y C y otro grupo de receptores, denominados receptores polimodales (PMRs), por su respuesta al estimulo mecánico y térmico en forma local (36).

Es ampliamente aceptado que la acupuntura genera la liberación de opioides endógenos entre estos las beta endorfinas que influyen en una variedad de funciones autónomas, percepción del dolor, respuesta al estrés y funciones inmunes; estos péptidos también pueden modular la respuesta inmune de algunas células tales como las $\mathrm{NK}$, debido a que ellas expresan receptores para este tipo de moléculas, demostrando la conexión existente entre el sistema nervioso y el sistema inmune $(36,37)$.

Estudios de imágenes diagnósticas en animales y humanos han mostrado que la EA activa el hipotálamo, centro primario de la modulación inmunoneuroendocrina, así como de actividades del sistema nervioso autónomo (37). Publicaciones de los últimos 20 años han mostrado que la acupuntura posiblemente afecta la síntesis y acción de varios neurotransmisores (catecolaminas, glutamato, acetilcolina, GABA y serotonina) y así como de neuropéptidos (oxitocina, sustancia P, entre otros) (36).

También se ha sugerido que las acciones de la acupuntura podrían ser mediadas por las neurotrofinas, familia de proteínas responsables del crecimiento y sobrevida neuronal durante su desarrollo, así como el mantenimiento y función de las neuronas adultas, por la modulación generada en el sistema nervioso central y periférico a través de la acupuntura (36). Localmente los puntos de acupuntura presentan mayor número de estructuras capilares, terminaciones nerviosas simpáticas, papilas térmicas y receptores así como un potencial eléctrico diferente con respecto a los puntos de piel ordinaria (39).

El microtrauma generado por las agujas inicia la secreción de histamina, bradiquinina, sustancia $\mathrm{P}$, serotonina entre otras, que conllevan a inflamación local. El Factor Hageman (factor XII), el plasminógeno, las quininas y la activación del sistema de complemento también forman parte, así como la hormona adrenocorticotropica (ACTH). $\mathrm{Su}$ secreción causa vasodilatación e incremento de la permeabilidad local, y como estos puntos poseen mayor número de terminaciones nerviosas, los efectos se amplifican generando migración local leucocitaria con secreción de citoquinas (factor de necrosis tumoral alfa (TNF- $\alpha$ ), IL-6, IL-1) que estimulan al hipotálamo en la secreción de CRH, activando el eje hipotálamo -hipófisis- adrenal que induce la secreción de glucocorticoides (cortisol) para regular la inflamación y los procesos de reparación (39).

\section{Posibles mecanismos reguladores de la acupuntura en la DA}

Evidencia emergente indica que la acupuntura estimula la inmunidad innata y la adquirida (39). Una serie de estudios, conducida por el Dr. Hisamitsu demostró que la EA sucesiva, estimulando el acupunto E36 (a la altura del borde distal de la tuberosidad anterior de la tibia) (Figuras 1) una vez al día por tres días, en ratones, incrementa la actividad de las NK en el bazo, células importantes en la respuesta inmune innata (37).

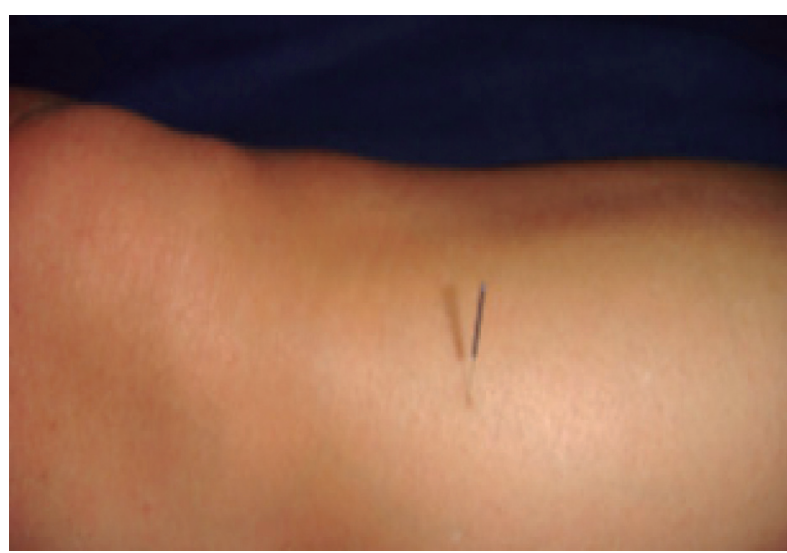

Figura 1. Representación de los puntos de acupuntura.

Otros estudios han indicado que la acupuntura o la EA pueden ser adecuados en el manejo de desordenes alérgicos (40), por su efecto en el desbalance Th1/Th2 como se evidenció en el estudio realizado por Park y col. (41) en ratones, a los que al inyectarles una proteína por via intraperitoneal, se les indujo una respuesta inmunológica mediada por linfocitos Th2 e IgE; luego se les realizó EA en el punto E36, dando como resultado una disminución significativa de los niveles séricos de IgE e IL4, citoquinas clásicas de la respuesta Th2 vista en la DA (41). Estos resultados fueron similares a los obtenidos en otros estudios realizados en pacientes con urticaria crónica y rinitis alérgica $(42,43)$.

En el estudio realizado por Yamaguchi y col. (44) se purificaron los leucocitos a partir de sangre periférica de 17 voluntarios sanos entre los 17 a 51 años antes y después del tratamiento con acupuntura, para determinar las variaciones de las células $T$, células $B$, macrófagos y NK. 
Los resultados mostraron un incremento en el conteo de las células $\mathrm{CD} 16^{+}, \mathrm{CD}^{2} 6^{+}$(marcadores asociados con la actividad de las $\mathrm{NK}$ ) y en el de las células $\mathrm{CD}^{+}, \mathrm{CD}^{+}$, $\mathrm{CD}^{+}$(marcadores asociados con la actividad de las células $\mathrm{T})$, en el día ocho después del tratamiento con acupuntura. Las células $\mathrm{CD} 19^{+}$(marcador asociado con la actividad de las células B), presentaron un incremento significativo, comparable con el observado en las células $\mathrm{CD} 16^{+}, \mathrm{CD} 56^{+}$. Los niveles de células CD11b ${ }^{+}$(marcador asociado con la actividad de los macrófagos), no mostraron un incremento significativo.

Para determinar si la estimulación con acupuntura podría afectar la maduración funcional de las células inmunocompetentes, se midieron por citometría los niveles de expresión de IL-1 $\beta$, IL-4 y IFN- $\gamma$, citoquinas asociadas con la actividad de los macrófagos y de la inmunidad humoral y celular. Los resultados mostraron que después del tratamiento había un incremento de los niveles de expresión de estas citoquinas. Con los resultados obtenidos los autores sugirieron que la acupuntura aumentaba la inmunidad celular y la humoral.

En el estudio realizado por Salameh y col. (45), en el cual participaron veinte pacientes entre los 13 a 48 años con diagnóstico de DA, quienes fueron tratados con acupuntura y medicina herbal China, recibieron dos sesiones de acupuntura semanal y formula herbal tres veces al día por un total de doce semanas. Para evaluar la eficacia del tratamiento administrado se utilizaron el Índice de calidad de vida en dermatología (DLQI), la escala análoga visual para prurito (VAS) y el Índice severidad y área de eczema (EASI). Al analizar los resultados se observó reducción en el VAS, DLQI y mejoría en el EASI.

Para demostrar el efecto que tiene la Moxibustión (acupuntura con calor), Takayama y col. (46) diseñaron un estudio en el que uso un modelo animal con infección por herpes tipo 1. Los animales fueron tratados con Moxibustión y se observó un incremento de la respuesta Th1 y producción de IFNg, lo que demostró que con el uso de este tratamiento se puede activar el sistema inmune para proteger contra la infección. Cheng y col. reportaron que con la utilización de moxa indirecta sobre el sitio de la lesión una vez al día por siete días en pacientes con DA, se observa una mejoría de la lesión en el 55\% de los pacientes (47). En el reporte de Boneberger y col. se realizó un manejo combinado de acupuntura y moxibustión en el tratamiento de eczema atópico. (puntos específicos: Xuehai, Quchi y Zhubin); la moxibustión sobre el sitio de la lesión, una vez al día por una semana. Después de dos semanas de finalizado el tratamiento se observó un 45\% de curación en el sitio de la lesión (48).

La regulación de la liberación de neuropéptidos en las terminaciones nerviosas inducida por la acupuntura puede tener importantes implicaciones dentro de la regulación de los procesos inflamatorios y manifestaciones sintomáticas de pacientes con procesos alérgicos (49). Inicialmente en el tratamiento con acupuntura se ha reportado actividad de la sustancia $P$, del neuropéptido $Y$, de péptidos intestinales vasoactivos, y de bradicinina así como de betaendorfinas, las cuales pueden incrementar la formación de citoquinas antiinflamatorias tales como IL-10 relacionada con las células T y/o disminución de citoquinas proinflamatorias (49).

Lee y col. (50) utilizando ratones, un modelo de dolor neuropático por cáncer, reportó que con el uso diario de EA sobre el punto E36 por 30 minutos durante nueve días, se inducía una disminución de la expresión de la sustancia P en el asta dorsal de la médula espinal, comparado con el grupo control, así como un incremento en la concentración de betaendorfina en sangre y cerebro de los ratones tratados. Los hallazgos anteriores sugieren la posible modulación de la acupuntura a nivel neuroendocrino.

En los últimos años tanto el cuerpo médico como los pacientes han visto la necesidad de buscar soluciones terapéuticas efectivas y con el menor número de efectos adversos para diversas patologías. Dentro de esta exploración, la acupuntura ha surgido como una opción que día a día toma mayor vigencia, tanto por el incremento del soporte científico que busca comprender los mecanismos neuroinmunoendocrinos involucrados en esta práctica ancestral, como por sus resultados terapéuticos. La DA, patología con un alto componente inmunológico, entraría dentro de este grupo de trastornos que podrían verse beneficiados con el uso de tratamientos complementarios como la acupuntura. Es por esto que se hace necesario promover un mayor número de estudios científicos que continúen explorando este campo que aunque es muy antiguo tiene gran proyección en el futuro.

\section{Referencias}

1. Burns T, Breathnach S, Cox N, Griffiths C, eds. Rooks Textbook of Dermatology, Vol. 1. Eighth Edition. Singapore: Wiley-Blackwell, 2010:24.1-24.31

2. Busi FM. Barrera epidérmica en dermatitis atópica. Rev Asoc Col Dermatol 2008; 16 (4): 293-301.

3. Williams HC. Atopic Dermatitis. N Engl J MED 2005; 352: 2314-2324.

4. Ballona R, Ballona C. Dermatitis Atópica: Revisión de tema. Folio Dermatol. 2004;15 (1):40-48 
5. Dennis R, Caraballo L, García E et al. Asthma and other allergic conditions in Colombia: a study in 6 cities. Ann Allergy Asthma Immunol. 2004; 93:568-574.

6. Worldwide variation in prevalence of symptoms of asthma, allergic rhinoconjunctivitis, and atopic eczema: ISAAC. The International Study of Asthma and Allergies in Childhood (ISAAC) Steering Committee. Lancet 1998; 351:1225-1232.

7. Lin YT, Wang CT, Chiang BL. Role of bacterial pathogens in atopic dermatitis. Clinic Rev Allerg Immunol 2007; 33: 167-177.

8. Bieber T. Atopic dermatitis. Ann Dermatol 2010; 22 (2): 125-137

9. Terui T. Analysis of the mechanism for the development of allergic skin inflammation and the application for its treatment: overview of the pathophysiology of atopic dermatitis. J Pharmacol Sci 2009; 110 (3): 232-236.

10. Elias PM, Steinhoff M. "Outside-to-inside" (and now back to "outside") pathogenic mechanisms in atopic dermatitis. J Invest Dermatol 2008; 128 (5): 1067-1070.

11. Gomes PL, Malavige GN, Fernando N, Mahendra MH, Kamaladasa SD, Seneviratne JK, et al. Characteristics of Staphylococcus aureus colonization in patients with atopic dermatitis in Sri Lanka. Clin Exp Dermatol 2011; 36 (2): 195-200.

12. aitanis G, Menounos P, Katsambas A, Velegraki A. Detection and mutation screening of Malassezia sympodialis sequences coding for the Mal s 1 allergen implicated in atopic dermatitis. J Invest Dermatol 2003; 121 (6): 1559-1560.

13. Friedman BC, Goldman RD. Anti-staphylococcal treatment in dermatitis. Can Fam Physician 2011; 57 (6): 669-671

14. Solanki LS, Srivastava N, Singh S. Superantigens: a brief review with special emphasis on dermatologic diseases. Dermatol Online J 2008; $14(2): 3$.

15. Ong PY, Leung DYM. The infectious aspects of atopic dermatitis. Immunol Allergy Clin North Am 2010; 30 (3): 309-321.

16. Kam L, Ben Chung L, Ping Chung L. Chinese herbal medicine research in eczema treatment. Hon et al. Chinese Medicine 2011, 6:17

17. Wollenberg A, Klein E. Current aspects of innata and adaptive immunity in atopic dermatitis. Clinic Rev Allerg Immunol 2007; 33: 35-44.

18. Novak N, Koch S, Allam JP, Bieber T. Dendritic cells: bridging innate and adpative immunity in atopic dermatitis. J Allergy Clin Immunol 2010; 125: 50-59.

19. Seneviratne SL, Black AP, Jones L, Bailey AS, Ogg GS. The role of skin-homing T cells in extrinsic atopic dermatitis. QJM 2007; 100 (1): 19-27

20. Gyimesi E, Nagy G, Remenyik E, Sipka S, Zeher M, Biro T, et al. Altered peripheral invariant natural killer $\mathrm{T}$ cells in atopic dermatitis. J Clin Immunol 2011. En prensa.

21. De Benedetto A, Agnihothri R, McGirt LY, Bankova LG, Beck LA. Atopic dermatitis: a disease caused by innate immune defects? J Invest Dermatol 2009; 129 (1): 14-30.

22. Bieber T, Novak Mecanismo Fisiopatologico de la dermatitis atopica. Allergy \& Clinical Immunol Inter 2005;17(1):26-29

23. Sio S, Puthia MK, Moochhala S, Bhatia M. The neuropeptide substance $\mathrm{P}$ is a critical mediator of burn-induced acute lung injury. J Immunol 2008; 180: 8333-8341.

24. Ohmura T, Tsunenari I, Hayashi T, Satoh $Y$, Konomi A, Nanri $H_{t}$ et al. Role of substance P in an NC/Nga mouse model of atopic dermatitis-like disease. Int Arch Allergy Immunol 2004; 133: 389-397.
25. ddor FA, Aoki V. Skin barrier in atopic dermatitis. An Bras Dermatol 2010; 85 (2): 184-194

26. Miller LS, Sørensen OE, Liu PT, Jalian HR, Eshtiaghpour D, Behmanesh BE, et al. TGF-alpha regulates TLR expression and function on epidermal keratinocytes. J Immunol 2005; 174 (10): 6137-6143.

27. Elias PM, Schmuth M. Abnormal skin barrier in the etiopathogenesis of atopic dermatitis. Curr Opin Allergy Clín Immunol 2009; 9 (5): 437-446. Hata TR, Gallo RL. Antimicrobial peptides, skin infections, and atopic dermatitis. Semin Cutan Med Surg 2008; 27 (2): 144-150.

28. Slattery MJ, Essex MJ, Paletz EM, Vanness ER, Infante M, Rogers GM, et al. Depression, anxiety, and dermatologic quality of life in adolescents with atopic dermatitis. J Allergy Clin Immunol 2011. En prensa.

29. Fernandez JM, Armario JC. Nuevas perspectivas terapéuticas en dermatitis atópica. Med Cutan Ibe Lat Am 2011;39( 1) : 30-36)

30. Anstey A. Management of atopic dermatitis: nonadherence to topical therapies in treatment of skin disease and the use of calcineurin inhibitors in difficult eczema. Br J Dermatol 2009; 161 (2): 219-220.

31. Aubert-Wastiaux H, Moret L, Le Rhun A, Fontenoy AM, Nguyen JM, Leux C, et al. Topical corticosteroid phobia in atopic dermatitis: a study of its nature, origins and frequency. Br J Dermatol 2011. En prensa.

32. McKenna SP, Doward LC. Quality of life of children with atopic dermatitis and their families. Curr Opin Allergy Clin Immunol 2008; 8 (3): 228-231

33. WHO International standard terminologies on tradicional medicine in the western pacific region who library cataloguing in publication data World Elath organization 2007

34. WHO Standart acupuncture point locations in the western pacific region who library cataloguing in publication data workd Elath organización 2008

35. Barrie C, Yyothirmai G. International Assocciacion for the study of pain Guia to pain Management in low resorce setting Chapter 9 Complementary therapies for pain Management .

36. Manni L, Albanesi M, Guaragna M, Barbaro S, Aloe L. Neurotrophins and acupuncture. Autonomic Neurosci 2010; 157: 9-17

37. Kim SK, Bae H. Acupuncture and immune modulation. Auton Neurosci 2010; 157: 38-41

38. Dhond RP, Yeh C, Park K, Kettner N, Napadow V. Acupuncture modulates resting state connectivity in default and sensorimotor brain networks. Pain 2008; 136 (3): 407-418

39. Cabioglu MT, Cetin BE. Acupuncture and immunomodulation. Am Chin Med 2008; 36 (1): 25-36.

40. Shiue S, Lee Y, Tsai C, Hsueh Y, Sheu J, Chang H.DNA microarray analysis of the effect on inflammation in patients treated with acupuncture for allergic rhinitis. J.Altern Complement Med 2008;14:689-698

41. Park MB, Ko E, Ahn C, Choi H, Rho S, Shin MK, et al. Suppression of $\operatorname{IgE}$ production and modulation of Th1/Th2 cell response by electroacupuncture in DNP-KLH immunized mice. J Neuroimmunol 2004; 151: 40-44.

42. Jianli C. The effect of acupuncture on serum IgE level in patients with chronic urticaria. J Tradit Chin Med 2006; 26 (3): 189-190.

43. Magnusson AL, Svensson REB, Leirvik C. The effect of acupuncture on allergic rhinitis: a randomized controlled clinical trial. Am I Chin Med 2004; 32 (1): 105-115. 
44. Yamaguchi N, Takahashi T, Sakuma M, Sugita T, Uchikawa K, Sakaihara S, et al. Acupuncture regulates leukocyte subpopulations in human peripheral blood. eCAM 2007; 4 (4): 447-453.

45. Salameh F, Perla D, Solomon M, Gamus D, Barzilai A, Greenberger $\mathrm{S}$, et al. The effectiveness of combined Chinese herbal medicine and acupuncture in the treatment of atopic dermatitis. J Altern Complement Med 2008; 14 (8): 1043-1048.

46. Takayama Y, Itoi M, Hamahashi T, Tsukamoto N, Mori K, Morishita $\mathrm{D}$, et al. Moxibustion activates host defense against herpes simplex virus type I through augmentation of cytokine production. Microbiol Immunol 2010; 54: 551-557.
47. Chung J, Hsin S. Acupuncture, electrostimulation and reflex therapy in dermatology. Dermatol Therapy 2003;16:87-92

48. Boneberger S, Rupec RA, Ruzicka T. Complementary therapy for atopic dermatitis and other allergic skin diseases: facts and controversies. Clin Dermatol 2010; 28: 57-61.

49. Zijlstra FJ, van den Berg-de Lange I, Huygen FJPM, Klein J. Antiinflammatory actions of acupuncture. Mediators of Inflammation 2003; 12 (2): 59-69.

50. Lee HJ, Lee JH, Lee EO, Lee HJK, Lee LS, Lee CH, et al. Substance P and beta endorphin mediate electroacupuncture induced analgesic activity in mouse cancer pain model. Acupunct Electro Ther Res 2009; 34: 27-40. 